\title{
On the Construction and Analysis of Finite Volume Element Schemes with Optimal $L^{2}$ Convergence Rate
}

\author{
Xiang Wang* and Yuqing Zhang \\ School of Mathematics, Jilin University, Changchun 130012, China \\ Received 16 February 2020; Accepted (in revised version) 12 May 2020
}

\begin{abstract}
We provide a general construction method for a finite volume element (FVE) scheme with the optimal $L^{2}$ convergence rate. The $k$-( $k$-1)-order orthogonal condition (generalized) is proved to be a sufficient and necessary condition for a $k$ order FVE scheme to have the optimal $L^{2}$ convergence rate in $1 \mathrm{D}$, in which the independent dual parameters constitute a $(k-1)$-dimension surface in the reasonable domain in $k$-dimension.

In the analysis, the dual strategies in different primary elements are not necessarily to be the same, and they are allowed to be asymmetric in each primary element, which open up more possibilities of the FVE schemes to be applied to some complex problems, such as the convection-dominated problems. It worth mentioning that, the construction can be extended to the quadrilateral meshes in 2D. The stability and $H^{1}$ estimate are proved for completeness. All the above results are demonstrated by numerical experiments.
\end{abstract}

AMS subject classifications: 65N12, 65N08, 65N30

Key words: Finite volume, $L^{2}$ estimate, sufficient and necessary condition, orthogonal condition.

\section{Introduction}

The finite volume element method (FVEM) $[1-3,6,9-11,14-16,18,19,21,26,29-$ $31,34,36]$ is a type of finite volume method (FVM), which is famous for the local conservation property and has been successfully applied to a broad range of problems. Till now, a lot of progress has been made in understanding the stability and $H^{1}$ estimate $[8,9,20,22,28,35,37], L^{2}$ estimate $[7,13,17,23,24,32]$, and superconvergence [4, $5,25,33$ ] for the FVEM. Most of the existing results talk about the FVE schemes with symmetric dual meshes.

\footnotetext{
${ }^{*}$ Corresponding author. Email addresses: wxjldx@jlu.edu.cn (X. Wang), 918809040@qq. com (Y. Zhang) 
This paper focuses on the construction and $L^{2}$ analysis for the FVE schemes whose dual meshes could be asymmetric in 1 dimension. Due to the test space of the FVEM is different from the trial space, given a Lagrange $k$-order trial space, there are different FVE schemes corresponding to the different choices of dual meshes and the piecewise constant test space over it. Nevertheless, not all of these FVE schemes have optimal $L^{2}$ convergence rate, including the even-order FVE schemes with uniform dual meshes. Therefore, how to choose the dual meshes is a main issue of the FVE schemes' construction [8, 23, 25, 32, 33, 35, 37]. To the authors' knowledge, current results about the $L^{2}$ estimate are mainly focused on sufficient conditions for FVE schemes to have the optimal $L^{2}$ convergence rate. At the same time, little progress has been made in understanding the necessary conditions.

In this paper, the $k$-(k-1)-order orthogonal condition (generalized) is proved to be a sufficient and necessary condition for the FVE schemes to hold the optimal $L^{2}$ convergence rate. The $k$ - $(k-1)$-order orthogonal condition means some orthogonality to the $(k-1)$-order polynomial space on each element $K$ in the sense of the inner product. It was first proposed in [32] for the FVE schemes with symmetric dual meshes over triangular meshes, and a simple comparative study was made on the number of independent restrictions and the number of independent dual parameters to determine the dual meshes. In the present paper, it is proved that, when the $k$ - $(k-1)$-order orthogonal condition is satisfied, the $k$ dual parameters form a $(k-1)$-dimension surface in the reasonable domain in $k$-dimension. That means, for the FVE schemes holding optimal $L^{2}$ convergence rate, their $k$ dual parameters form a $(k$-1)-dimension surface in $k$-dimension. In particular, for FVE schemes with symmetric dual meshes, all odd-order schemes have optimal $L^{2}$ convergence rate, while not all even-order schemes hold optimal $L^{2}$ convergence rate even if they have uniform dual meshes, which are consistent with what we knew before from numerical experiments.

It's worth mentioning that, in the analysis, the dual strategies in different element could be different, which allows more applications of the FVEM. Numerical experiments are presented for some convection-dominated problems (Examples 5.4-5.5). The performances of the standard quadratic finite element (FE) scheme (FE2), the quadratic FVE scheme with Gauss-Lobatto dual strategy (FV2-5), and the quadratic FVE schemes (FV2-6 in Example 5.4 and FV2-7 in Example 5.5) with asymmetric dual meshes are compared over uniform primary meshes. It's shown that the quadratic FVE schemes (FV2-6 and FV2-7) with proper dual strategies perform better than FE2 and FV2-5 in these two examples, which demonstrates the capacity of the FVEM to solve some convection-dominated problems.

Following Section 2 shows the definition of the FVE schemes for the two-points boundary value problem. Section 3 introduces the orthogonal condition and the corresponding equivalent equations, which helps to construct FVE schemes. In Section 4, we present the main result of this paper, a necessary and sufficient condition for a FVE scheme to have the optimal $L^{2}$ convergence rate. Numerical experiments, including one on the rectangular meshes, are shown in Section 5 to illustrate our theoretical results. Then, we made conclusion in Section 6 and analyse the stability in Appendix A. 


\section{FVE schemes of arbitrary order}

Consider the two-point boundary value problem,

$$
\left\{\begin{array}{l}
-\left(p(x) u^{\prime}(x)\right)^{\prime}+q(x) u^{\prime}(x)+r(x) u(x)=f(x), \quad \forall x \in \Omega:=(0,1), \\
u(0)=u(1)=0
\end{array}\right.
$$

where

$$
p \geq p_{0}>0, \quad r-\frac{1}{2} q^{\prime} \geq \gamma>0, \quad p, q, r \in L^{\infty}, \quad f \in L^{2}(\Omega), \quad\|p\|_{2, \infty} \leq \overline{p_{2}} .
$$

\subsection{The trial function space and test function space}

Primary mesh and trial function space. Let $0=x_{0}<x_{1}<x_{2}<\cdots<x_{N}=1$ be $N+1$ distinct points on $\bar{\Omega}$. For all $i \in \mathbb{Z}_{N}:=\{1, \ldots, N\}$, we denote $K_{i}=\left[x_{i-1}, x_{i}\right]$ and $h_{i}=x_{i}-x_{i-1}$. Let $h=\max _{i \in \mathbb{Z}_{N}} h_{i}$ and

$$
\mathcal{T}_{h}=\left\{K_{i}: i \in \mathbb{Z}_{N}\right\}
$$

be a partition (primary mesh) of $\Omega$. The corresponding trial function space $U_{h}^{k}$ is chosen as the $k$-order $(k \geq 1)$ Lagrange finite element space

$$
U_{h}^{k}:=\left\{w_{h} \in C(\Omega):\left.w_{h}\right|_{K} \in P^{k}(K), \forall K \in \mathcal{T}_{h},\left.w_{h}\right|_{\partial \Omega}=0\right\} .
$$

Here, $P^{k}(K)$ is the $k$-order polynomial space on $K$. It's easy to find that $\operatorname{dim} U_{h}^{k}=$ $N k-1$.

Dual mesh and test function space. Let $0<\alpha_{1}<\alpha_{2}<\cdots<\alpha_{k}<1$ be $k$ points (to define the dual points) on the reference interval $\hat{K}=[0,1]$. The dual points on each interval $K_{i}\left(i \in \mathbb{Z}_{N}\right)$ are defined as the affine transformations of $\alpha_{j}$ s from $\hat{K}$ to $K_{i}$, that

$$
\alpha_{i, j}=h_{i} \alpha_{j}+x_{i-1}, \quad(i, j) \in \mathbb{Z}_{N} \times \mathbb{Z}_{k}, \quad \alpha_{N, k+1}=1 .
$$

With these dual points, we construct the dual meshes

$$
\mathcal{T}_{h}^{*}=\left\{K_{1,0}^{*}\right\} \cup\left\{K_{i, j}^{*}:(i, j) \in \mathbb{Z}_{N} \times \mathbb{Z}_{k}\right\},
$$

where

$$
K_{1,0}^{*}=\left[0, \alpha_{1,1}\right], \quad K_{i, j}^{*}=\left[\alpha_{i, j}, \alpha_{i, j+1}\right], \quad \alpha_{i, k+1}=\alpha_{i+1,1}, \quad \forall i \in \mathbb{Z}_{N-1} .
$$

The corresponding test function space $V_{h}$ is the piecewise constant function space over $\mathcal{T}_{h}^{*}$, which vanishes on the intervals $K_{1,0}^{*} \cup K_{N, k}^{*}$

$$
V_{h}:=\left\{v_{h}: v_{h}=\sum_{i=1}^{N} \sum_{j=1}^{k} v_{i, j} \psi_{i, j},(i, j) \in \mathbb{Z}_{N} \times \mathbb{Z}_{k}, v_{1,0}=v_{N, k}=0\right\},
$$

where $v_{i, j}$ and $\psi_{i, j}=\chi\left[\alpha_{i, j}, \alpha_{i, j+1}\right]$ are the constant function and the characteristic function on $K_{i, j}^{*}$, respectively. Here, we have $\operatorname{dim} V_{h}=N k-1=\operatorname{dim} U_{h}^{k}$. 


\subsection{FVE schemes}

Integrating (2.1) on each control volume $K_{i, j}^{*}=\left[\alpha_{i, j}, \alpha_{i, j+1}\right] \in \mathcal{T}_{h}^{*}$ with integration by parts, we have

$$
\begin{aligned}
p\left(\alpha_{i, j}\right) & u^{\prime}\left(\alpha_{i, j}\right)-p\left(\alpha_{i, j+1}\right) u^{\prime}\left(\alpha_{i, j+1}\right) \\
& +\int_{\alpha_{i, j}}^{\alpha_{i, j+1}} q(x) u^{\prime}(x)+r(x) u(x) d x=\int_{\alpha_{i, j}}^{\alpha_{i, j+1}} f(x) d x .
\end{aligned}
$$

For any $v_{h} \in V_{h}$, multiplying (2.2) with $v_{i, j}$ and the summing up for all $K_{i, j}^{*} \in \mathcal{T}_{h}^{*}$, approximate $u$ in the trial space $U_{h}^{k}$. Then, the FVE scheme for solving (2.1) is to find $u_{h} \in U_{h}^{k}$, such that

$$
a_{h}\left(u_{h}, v_{h}\right)=\left(f, v_{h}\right), \quad \forall v_{h} \in V_{h},
$$

where $\left(f, v_{h}\right)$ is the normal inner product, and $a_{h}\left(u_{h}, v_{h}\right)$ is the bilinear form of the FVEM

$$
\begin{aligned}
a_{h}\left(u_{h}, v_{h}\right)=\sum_{K_{i} \in \mathcal{T}_{h}} & \sum_{K_{i, j}^{*} \in \mathcal{T}_{h}^{*}}-\left.\left(p u_{h}^{\prime} v_{h}\right)\right|_{\partial K_{i, j}^{*} \cap K_{i}} \\
& +\sum_{K_{i} \in \mathcal{T}_{h}} \int_{K_{i}} v_{h}\left(q(x) u_{h}^{\prime}(x)+r(x) u_{h}(x)\right) d x
\end{aligned}
$$

which also can be written as

$$
a_{h}\left(u_{h}, v_{h}\right)=\sum_{i=1}^{N} \sum_{j=1}^{k}\left[v_{i, j}\right] p\left(\alpha_{i, j}\right) u_{h}^{\prime}\left(\alpha_{i, j}\right)+\sum_{i=1}^{N} \sum_{j=1}^{k} v_{i, j} \int_{\alpha_{i, j}}^{\alpha_{i, j+1}} q(x) u_{h}^{\prime}(x)+r(x) u_{h}(x) d x .
$$

Here, $\left[v_{i, j}\right]=v_{i, j}-v_{i, j-1}$ is the jump of $v_{h}$ at point $\alpha_{i, j}$, and $v_{i, 0}=v_{i-1, k}, 2 \leq i \leq N$.

\subsection{Notations about interpolation operators}

To the convenience of the proof in this paper, we define the following two operators $\Pi_{h}^{k}$ and $\Pi_{h}^{k, *}$.

- $\Pi_{h}^{k}: H^{1}(\Omega) \rightarrow U_{h}^{k}$, the piecewise $k$-order Lagrange interpolation operator.

- $\Pi_{h}^{k, *}: U_{h}^{k} \rightarrow V_{h}$, a piecewise constant operator based on the dual mesh $\mathcal{T}_{h}^{*}$.

Let $0=a_{0}<a_{1}<a_{2}<\cdots<a_{k}=1$ be $k+1$ points on the reference interval $\hat{K}=[0,1]$. Then, define the interpolation nodes of $\Pi_{h}^{k, *}$ on $K_{i}$ by the affine transformations of $a_{j} \mathrm{~s}$ from $\hat{K}$ to $K_{i}$, that

$$
a_{1,0}=0, \quad a_{i, j}=x_{i-1}+h_{i} a_{j}, \quad(i, j) \in \mathbb{Z}_{N} \times \mathbb{Z}_{k} .
$$

Then, for any $w_{h} \in U_{h}^{k}, \Pi_{h}^{k, *} w_{h}$ is given by

$$
\left.\Pi_{h}^{k, *} w_{h}\right|_{K_{i, j}^{*}}=w_{h}\left(a_{i, j}\right), \quad \forall K_{i, j}^{*} \in \mathcal{T}_{h}^{*} .
$$




\section{The orthogonal condition}

The orthogonal condition for the $k$-order FVEM with general dual strategies are given by

Definition 3.1 (The $k$-( $k$-1)-order orthogonal condition). We call a $k$-order FVE scheme satisfying the $k$ - $(k-1)$-order orthogonal condition, if there exists $\Pi_{h}^{k, *}$, such that the following equations (constraints) hold

$$
\int_{K} g\left(w-\Pi_{h}^{k, *} w\right) d x=0, \quad \forall g \in P^{k-1}(K), \quad \forall w \in P^{1}(K) .
$$

Here, $P^{k-1}(K)$ is the $(k-1)$-order polynomial space on $K$.

Lemma 3.1. The $k$-(k-1)-order orthogonal condition (3.1) is equivalent to following restrictions

$$
\sum_{j=1}^{k}\left(a_{j}-a_{j-1}\right) \alpha_{j}^{i}=\frac{1}{i+1}, \quad \forall i \in Z_{k} .
$$

Here, $\alpha_{j} s\left(j \in \mathbb{Z}_{k}\right)$ and $a_{i} s\left(i \in \mathbb{Z}_{k} \cup\{0\}\right)$ are the parameters to locate the dual points and the interpolation nodes of $\Pi_{h}^{k, *}$, respectively, which are defined in Subsections 2.1 and 2.3.

Proof. Consider the interpolation $\hat{\Pi}_{h}^{k, *}$ on the reference element $\hat{K}=[0,1]$. Notice that when $w$ is a constant on $\hat{K}, w \equiv \hat{\Pi}_{h}^{k, *} w$. That means (3.1) hold when $w$ is a constant. Thus, with the facts that $P^{k-1}([0,1])=\operatorname{Span}\left\{1, x, \ldots, x^{k-1}\right\},(3.1)$ is equivalent to

$$
\int_{0}^{1} g\left(x-\hat{\Pi}_{h}^{k, *} x\right) d x=0, \quad \forall g \in\left\{1, x, \ldots, x^{k-1}\right\} .
$$

We further arrive

$$
\int_{0}^{1} x^{i} \hat{\Pi}_{h}^{k, *} x d x=\int_{0}^{1} x^{i+1} d x, \quad \forall i \in\{0,1, \ldots, k-1\}
$$

which leads to

$$
\int_{0}^{1} x^{i} \hat{\Pi}_{h}^{k, *} x d x=\frac{1}{i+2}, \quad \forall i \in\{0,1, \ldots, k-1\} .
$$

Substitute the expression of $\hat{\Pi}_{h}^{k, *}$ into (3.4) then we have

$$
\frac{1}{i+1}\left(\sum_{j=1}^{k-1} a_{j}\left(\alpha_{j+1}^{i+1}-\alpha_{j}^{i+1}\right)+\left(1-\alpha_{k}^{i+1}\right)\right)=\frac{1}{i+2}, \quad \forall i \in\{0,1, \ldots, k-1\} .
$$

Through a simple calculation, we have the conclusion of Lemma 3.1.

Lemma 3.2. Given $k$, there exists an operator $\Pi_{h}^{k, *}$, such that the $k$-(k-1)-order orthogonal condition is satisfied. 
Proof. Recalling (3.3), with a simple calculation, the $k$-(k-1)-order orthogonal condition is equivalent to the following restrictions

$$
\sum_{j=1}^{k}\left(a_{j}-a_{j-1}\right) \alpha_{j}^{i+1}=\int_{0}^{1} x^{i+1} d x, \quad \forall i=0,1, \ldots, k-1 .
$$

Summing the coefficients of $\alpha_{j}^{i+1} s$, we have

$$
\sum_{j=1}^{k}\left(a_{j}-a_{j-1}\right)=a_{k}-a_{0}=1-0=1 .
$$

Thus, the coefficients $\left(a_{j}-a_{j-1}\right)$ in (3.5) could be the weights of the $k$-points numerical quadrature corresponding to $\alpha_{j} s$. Since a $k$-points integration rule is accurate for $(k$ 1)-order polynomials (see, e.g., [12]), the $k$-( $k$-1)-order orthogonal condition could be satisfied with a proper selection of $\alpha_{j}$ s and $a_{j}$ s. Which ends the proof of Lemma 3.2.

Lemma 3.3. Let $\mathbf{x}_{t}=\left(x_{1}, x_{2}, \ldots, x_{t}\right)_{t \times 1}^{T}$, satisfying $0<x_{1}<\cdots<x_{t}<1$. Then,

$$
M\left(\mathbf{x}_{t}\right)=\left(\begin{array}{cccc}
x_{1} & x_{2} & \cdots & x_{t} \\
x_{1}^{2} & x_{2}^{2} & \cdots & x_{t}^{2} \\
\cdots & \cdots & \cdots & \cdots \\
x_{1}^{t} & x_{2}^{t} & \cdots & x_{t}^{t}
\end{array}\right)_{t \times t}
$$

is an irreducible (invertible) Vandermonde matrix.

Proof. The determinant of the Vandermonde matrix $M\left(\mathrm{x}_{t}\right)$ can be easily given by

$$
\operatorname{det}\left(M\left(\mathbf{x}_{t}\right)\right)=\prod_{i=1}^{t} x_{i} \prod_{1 \leq j<s \leq t}\left(x_{s}-x_{j}\right) .
$$

Since $0<x_{1}<x_{2}<\cdots<x_{t}<1$, we have

$$
x_{s}-x_{j}<0, \quad \forall 1 \leq s \leq j .
$$

Thus,

$$
\operatorname{det}\left(M\left(\mathbf{x}_{t}\right)\right) \neq 0
$$

Thus, $M\left(\mathbf{x}_{t}\right)$ is invertible.

Now, we are ready to present when will a dual strategy on $K$ satisfy the $k$-(k-1)order orthogonal condition for the $k$-order FVEM.

Lemma 3.4. Let $\mathcal{D}_{\alpha, k}:=\left\{\left(\alpha_{1}, \ldots, \alpha_{k}\right)_{1 \times k}^{T}: 0<\alpha_{1}<\cdots<\alpha_{k}<1\right\}$ be the reasonable domain of the parameters $\vec{\alpha}_{k}$, which determine the dual strategy on $K$ for the k-order FVE schemes. Then, the solutions $\vec{\alpha}_{k} \in \mathcal{D}_{\alpha, k}$ of the $k$-(k-1)-order orthogonal condition (3.1) form a ( $k$-1)-dimension surface in $\mathcal{D}_{\alpha, k}$, which is in $k$-dimension. 
Proof. From (3.2), the constraints of (3.1) are equivalent to a linear algebra

$$
M\left(\vec{\alpha}_{k}\right) C \mathbf{a}=\mathbf{b},
$$

where $M\left(\vec{\alpha}_{k}\right)$ is defined by (3.6), and

$$
C=\left(\begin{array}{rrrrc}
1 & 0 & 0 & \cdots & 0 \\
-1 & 1 & 0 & \cdots & 0 \\
0 & -1 & 1 & \cdots & 0 \\
\vdots & \vdots & \vdots & \vdots & \vdots \\
0 & 0 & 0 & \cdots & 1
\end{array}\right)_{k \times k}, \quad \mathbf{a}=\left(\begin{array}{c}
a_{1} \\
a_{2} \\
a_{3} \\
\vdots \\
a_{k}
\end{array}\right)_{k \times 1}, \quad \mathbf{b}=\left(\begin{array}{c}
1 / 2 \\
1 / 3 \\
1 / 4 \\
\vdots \\
1 /(k+1)
\end{array}\right)_{k \times 1}
$$

with $a_{k} \equiv 1$. Here, $M\left(\vec{\alpha}_{k}\right)$ is invertible (from Lemma 3.3) and $C$ is obviously invertible. For a given $\vec{\alpha}_{k} \in \mathcal{D}_{\alpha, k}$, it's impossible to always have a reasonable a such that

$$
\mathbf{a}=C^{-1}\left(M\left(\vec{\alpha}_{k}\right)\right)^{-1} \mathbf{b}
$$

since the last component $a_{k}$ is fixed as $a_{k}=1$.

However, with Lemma 3.2, there always exist appropriate $\vec{\alpha}_{k}$ and a for (3.7) to be true. Considering $\vec{\alpha}_{k}$ as parameter equations of a. It's easy to see that the reasonable $\vec{\alpha}_{k}$ from (3.7) form a ( $k$-1)-dimension surface in the $k$-dimension domain $\mathcal{D}_{\alpha, k}$. Which ends the proof of conclusion in Lemma 3.4.

\subsection{Equivalent equations for $k=2,3,4$}

Here, we show the equivalent equations of the $k$-(k-1)-order orthogonal condition for $k \leq 4$.

- For $k=1$, the equivalent equations of (3.1) lead to the unique solution $\alpha_{1}=\frac{1}{2}$.

- For $k=2$, the equivalent equations of (3.1) are

$$
\left\{\begin{array}{l}
a_{1} \alpha_{1}+\left(1-a_{1}\right) \alpha_{2}=\frac{1}{2}, \\
a_{1} \alpha_{1}^{2}+\left(1-a_{1}\right) \alpha_{2}^{2}=\frac{1}{3},
\end{array}\right.
$$

where $0<a_{1}<1$ and $0<\alpha_{1}<\alpha_{2}<1$. Write $\alpha_{1}$ and $\alpha_{2}$ as parameter functions of $a_{1}$. Thus,

$$
\left\{\begin{array}{l}
\alpha_{1}=\frac{1}{2}-\frac{1-a_{1}}{a_{1}} \sqrt{\frac{a_{1}}{12\left(1-a_{1}\right)}}, \\
\alpha_{2}=\frac{1}{2}+\sqrt{\frac{a_{1}}{12\left(1-a_{1}\right)}},
\end{array}\right.
$$

where $\frac{1}{4}<a_{1}<\frac{3}{4}$. 
- For $k=3$, the equivalent equations of (3.1) are

$$
\left\{\begin{array}{l}
a_{1} \alpha_{1}+\left(a_{2}-a_{1}\right) \alpha_{2}+\left(1-a_{2}\right) \alpha_{3}=\frac{1}{2}, \\
a_{1} \alpha_{1}^{2}+\left(a_{2}-a_{1}\right) \alpha_{2}^{2}+\left(1-a_{2}\right) \alpha_{3}^{2}=\frac{1}{3}, \\
a_{1} \alpha_{1}^{3}+\left(a_{2}-a_{1}\right) \alpha_{2}^{3}+\left(1-a_{2}\right) \alpha_{3}^{3}=\frac{1}{4},
\end{array}\right.
$$

where $0<a_{1}<a_{2}<1$ and $0<\alpha_{1}<\alpha_{2}<1$.

- For $k=4$, the equivalent equations of (3.1) are

$$
\left\{\begin{array}{l}
a_{1} \alpha_{1}+\left(a_{2}-a_{1}\right) \alpha_{2}+\left(a_{3}-a_{2}\right) \alpha_{3}+\left(1-a_{3}\right) \alpha_{4}=\frac{1}{2} \\
a_{1} \alpha_{1}^{2}+\left(a_{2}-a_{1}\right) \alpha_{2}^{2}+\left(a_{3}-a_{2}\right) \alpha_{3}^{2}+\left(1-a_{3}\right) \alpha_{4}^{2}=\frac{1}{3} \\
a_{1} \alpha_{1}^{3}+\left(a_{2}-a_{1}\right) \alpha_{2}^{3}+\left(a_{3}-a_{2}\right) \alpha_{3}^{3}+\left(1-a_{3}\right) \alpha_{4}^{3}=\frac{1}{4}, \\
a_{1} \alpha_{1}^{4}+\left(a_{2}-a_{1}\right) \alpha_{2}^{4}+\left(a_{3}-a_{2}\right) \alpha_{3}^{4}+\left(1-a_{3}\right) \alpha_{4}^{4}=\frac{1}{5},
\end{array}\right.
$$

where $0<a_{1}<a_{2}<a_{3}<1$ and $0<\alpha_{1}<\alpha_{2}<\alpha_{3}<1$.

Here, we show in Fig. 1 the reasonable solutions of the orthogonal condition for $k=2,3$, which are a 1-dimension line and a 2-dimension surface in the 2-dimension and 3-dimension domains, respectively.

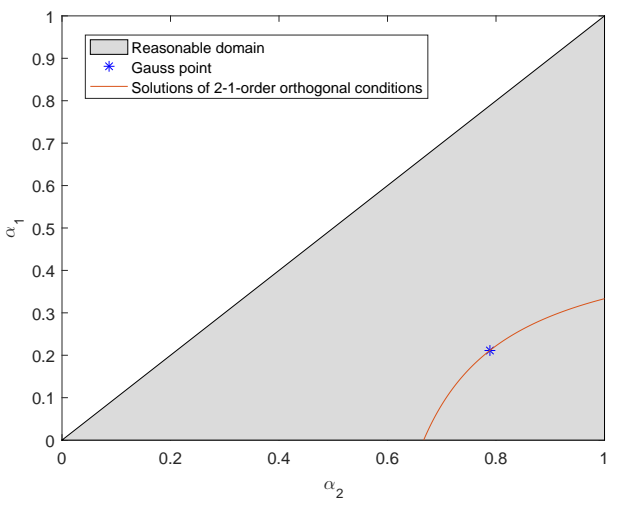

a) $k=2$

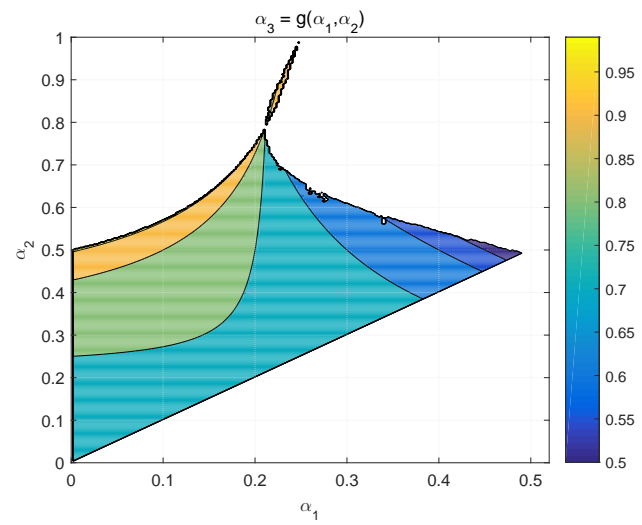

b) $k=3$

Figure 1: The parameters satisfy the $k$-(k-1)-order orthogonal condition.

\section{4. $L^{2}$ estimate}

We prove that the $k$-(k-1)-order orthogonal condition is a sufficient and necessary condition for a $k$-order FVE scheme to have optimal $L^{2}$ convergence rate. The stability 
and $H^{1}$ estimate, which are the basis of $L^{2}$ convergence, will be proved later in Appendix A, in which Theorem A.2 shows the $H^{1}$ estimate for the finite volume schemes that

$$
\left\|u-u_{h}\right\|_{1} \leq C h^{k}\|u\|_{k+1}
$$

under the assumption $u \in H_{0}^{1}(\Omega) \cap H_{\mathcal{T}}^{k+1}(\Omega)$.

\subsection{The sufficiency}

Theorem 4.1. Suppose $u \in H_{0}^{1}(\Omega) \cap H^{k+2}(\Omega)$ is the exact solution of (2.1), $\mathcal{T}_{h}$ is regular. For a k-order Lagrange trial function space $U_{h}^{k}$, choose $\mathcal{T}_{h}^{*}$ satisfying the $k$-(k-1)-order orthogonal condition. Let $u_{h} \in U_{h}^{k}$ be the solution of the corresponding k-order FVE scheme (2.3). Then, there exists a positive constant $C$ such that

$$
\left\|u-u_{h}\right\|_{0} \leq C h^{k+1}\|u\|_{k+2}
$$

Proof. We begin with the Aubin-Nitsche technique. The auxiliary problem is given by: For any $g \in L^{2}(\Omega)$, find $w \in H_{0}^{1}(\Omega)$ such that

$$
a(v, w)=(g, v), \quad \forall v \in H_{0}^{1}(\Omega),
$$

where

$$
a(v, w)=\int_{\Omega} p v^{\prime} w^{\prime}+\left(q v^{\prime}+r v\right) w d x, \quad(g, v)=\int_{\Omega} g v d x .
$$

Clearly, from the regularity of the auxiliary problem (4.3), we have $w \in H_{0}^{1}(\Omega) \cap H^{2}(\Omega)$ and some constant $C$ such that,

$$
\|w\|_{2} \leq C\|g\|_{0} .
$$

Let $v=g=u-u_{h}$ in (4.3). Notice the orthogonality of FVE solution that

$$
a_{h}\left(u-u_{h}, \Pi_{h}^{k, *}\left(\Pi_{h}^{1} w\right)\right)=0,
$$

then

$$
\begin{aligned}
& \left\|u-u_{h}\right\|_{0}^{2}=a\left(u-u_{h}, w\right) \\
= & a\left(u-u_{h}, w-\Pi_{h}^{1} w\right)+a\left(u-u_{h}, \Pi_{h}^{1} w\right)-a_{h}\left(u-u_{h}, \Pi_{h}^{k, *}\left(\Pi_{h}^{1} w\right)\right) .
\end{aligned}
$$

For the first term on the right-hand side of (4.5) with (4.1) and the estimate of the linear interpolations we have

$$
\begin{aligned}
a\left(u-u_{h}, w-\Pi_{h}^{1} w\right) & \leq C\left\|u-u_{h}\right\|_{1}\left\|w-\Pi_{h}^{1} w\right\|_{1} \\
& \leq C h^{k+1}\|u\|_{k+1}\|w\|_{2} .
\end{aligned}
$$


Following, we estimate the difference between $a\left(u-u_{h}, \Pi_{h}^{1} w\right)$ and $a_{h}\left(u-u_{h}\right.$, $\left.\Pi_{h}^{k, *}\left(\Pi_{h}^{1} w\right)\right)$. With the integration by parts, the diffusion terms of the bilinear forms can be rewritten by

$$
\begin{aligned}
& \sum_{K \in \mathcal{T}_{h}} \int_{K} p\left(u-u_{h}\right)^{\prime}\left(\Pi_{h}^{1} w\right)^{\prime} d x \\
= & \left.\sum_{K \in \mathcal{T}_{h}}\left(p\left(u-u_{h}\right)^{\prime} \Pi_{h}^{1} w\right)\right|_{\partial K}-\sum_{K \in \mathcal{T}_{h}} \int_{K}\left(p\left(u-u_{h}\right)^{\prime}\right)^{\prime}\left(\Pi_{h}^{1} w\right) d x, \\
& \sum_{K \in \mathcal{T}_{h}} \sum_{K^{*} \in \mathcal{T}_{h}^{*}}-\left.\left(p\left(u-u_{h}\right)^{\prime} \Pi_{h}^{k, *}\left(\Pi_{h}^{1} w\right)\right)\right|_{\partial K^{*} \cap K} \\
= & \left.\sum_{K \in \mathcal{T}_{h}}\left(p\left(u-u_{h}\right)^{\prime} \Pi_{h}^{k, *}\left(\Pi_{h}^{1} w\right)\right)\right|_{\partial K}-\sum_{K \in \mathcal{T}_{h}} \int_{K}\left(p\left(u-u_{h}\right)^{\prime}\right)^{\prime} \Pi_{h}^{k, *}\left(\Pi_{h}^{1} w\right) d x .
\end{aligned}
$$

Notice that $\left.\Pi_{h}^{1} w\right|_{\partial K}=\left.\Pi_{h}^{k, *}\left(\Pi_{h}^{1} w\right)\right|_{\partial K}$, then, the first terms on the right-hand side of (4.7) and (4.8) are equal that

$$
\left.\sum_{K \in \mathcal{T}_{h}}\left(p\left(u-u_{h}\right)^{\prime} \Pi_{h}^{1} w\right)\right|_{\partial K}=\left.\sum_{K \in \mathcal{T}_{h}}\left(p\left(u-u_{h}\right)^{\prime} \Pi_{h}^{k, *}\left(\Pi_{h}^{1} w\right)\right)\right|_{\partial K}
$$

Since the orthogonal condition (3.1) is satisfied, with the fact that $\Pi_{h}^{k-1} u_{h}^{\prime}=u_{h}^{\prime} \in$ $P^{k-1}\left(K_{i}\right)$, we have

$$
\begin{aligned}
& \quad\left|\sum_{K \in \mathcal{T}_{h}} \int_{K} p\left(u-u_{h}\right)^{\prime}\left(\Pi_{h}^{1} w\right)^{\prime} d x-\sum_{K \in \mathcal{T}_{h}} \sum_{K^{*} \in \mathcal{T}_{h}^{*}}-\left(p\left(u-u_{h}\right)^{\prime} \Pi_{h}^{k, *}\left(\Pi_{h}^{1} w\right)\right)\right|_{\partial K^{*} \cap K} \mid \\
& =\left|\sum_{K \in \mathcal{T}_{h}} \int_{K}\left(\left(p-p_{0, K}+p_{0, K}\right)(u-u h)^{\prime}\right)^{\prime}\left(\Pi_{h}^{1} w-\Pi_{h}^{k, *}\left(\Pi_{h}^{1} w\right)\right) d x\right| \\
& \leq\left|\sum_{K \in \mathcal{T}_{h}} \int_{K}\left(\left(p-p_{0, K}\right)\left(u-u_{h}\right)^{\prime}\right)^{\prime}\left(\Pi_{h}^{1} w-\Pi_{h}^{k, *}\left(\Pi_{h}^{1} w\right)\right) d x\right| \\
& \quad+\left|\sum_{K \in \mathcal{T}_{h}} p_{0, K} \int_{K}\left(\left(u-u_{h}\right)^{\prime \prime}-\Pi_{h}^{k-1}\left(u-u_{h}\right)^{\prime \prime}\right)\left(\Pi_{h}^{1} w-\Pi_{h}^{k, *}\left(\Pi_{h}^{1} w\right)\right) d x\right| \\
& \leq C h\|p\|_{2, \infty}\left\|u-u_{h}\right\|_{2}\left\|\Pi_{h}^{1} w-\Pi_{h}^{k, *}\left(\Pi_{h}^{1} w\right)\right\|_{0} \\
& \quad+\left|\sum_{K \in \mathcal{T}_{h}} \int_{K} p_{0, K}\left(u^{\prime \prime}-\Pi_{h}^{k-1} u^{\prime \prime}\right)\left(\Pi_{h}^{1} w-\Pi_{h}^{k, *}\left(\Pi_{h}^{1} w\right)\right) d x\right|
\end{aligned}
$$

(with the orthogonal condition)

$\leq C h^{2}\|p\|_{2, \infty}\left\|u-u_{h}\right\|_{2}\|w\|_{1}+C\|p\|_{0, \infty} h^{k+1}\|u\|_{k+2}\|w\|_{1}$

$\lesssim h^{k+1}\|u\|_{k+2}\|w\|_{1}$. 
Here, $p_{0, K}$ is the average of $p$ on $K$. Thus,

$$
\begin{aligned}
& \left|a\left(u-u_{h}, \Pi_{h}^{1} w\right)-a_{h}\left(u-u_{h}, \Pi_{h}^{k, *}\left(\Pi_{h}^{1} w\right)\right)\right| \\
= & \mid-\sum_{K \in \mathcal{T}_{h}} \int_{K}\left(p\left(u-u_{h}\right)^{\prime}\right)^{\prime}\left(\Pi_{h}^{1} w-\Pi_{h}^{k, *}\left(\Pi_{h}^{1} w\right)\right) d x \\
& \quad+\sum_{K \in \mathcal{T}_{h}} \int_{K}\left(q\left(u-u_{h}\right)^{\prime}+r\left(u-u_{h}\right)\right)\left(\Pi_{h}^{1} w-\Pi_{h}^{k, *}\left(\Pi_{h}^{1} w\right)\right) d x \mid \\
\leq & C h^{k+1}\|u\|_{k+2}\|w\|_{1}+C\left\|u-u_{h}\right\|_{1}\left\|\Pi_{h}^{1} w-\Pi_{h}^{k, *}\left(\Pi_{h}^{1} w\right)\right\|_{0} \\
\leq & C h^{k+1}\|u\|_{k+2}\|w\|_{1} .
\end{aligned}
$$

From (4.4)-(4.6) and (4.10), one can conclude that

$$
\begin{aligned}
\left\|u-u_{h}\right\|_{0}^{2} & \leq C h^{k+1}\|u\|_{k+1}\|w\|_{2}+C h^{k+1}\|u\|_{k+2}\|w\|_{1} \\
& \leq C h^{k+1}\|u\|_{k+2}\|w\|_{2} \\
& \leq C h^{k+1}\|u\|_{k+2}\left\|u-u_{h}\right\|_{0}
\end{aligned}
$$

Eliminate $\left\|u-u_{h}\right\|_{0}$, we have the optimal $L^{2}$ estimate (4.2).

\subsection{The necessity}

To prove the necessity, one should prove that all the FVE schemes, which do not satisfy the $k$ - $(k-1)$-order orthogonal condition, can not reach the optimal $L^{2}$ convergence rate. However, the optimal $L^{2}$ estimate (4.2) is an inequality itself, and there are many inequality estimates in the proof. All these make the proof of necessity much harder than sufficiency.

Theorem 4.2. A $k$-order FVE scheme to solve (2.1) will hold the optimal $L^{2}$ convergence rate if and only if the $k$-(k-1)-order orthogonal condition is satisfied.

Proof. The sufficiency is given by Theorem 4.1. Following, we analysis the necessity: for any FVE scheme, if the $k$-(k-1)-order orthogonal condition is not satisfied, there exist $u$ and $u_{h}$ such that

$$
\left\|u-u_{h}\right\|_{0} \geq C h^{k}\|u\|_{k+1}
$$

Here, $C$ is independent of $h$.

From the proof of Lemma 3.4, we can find that, any $k$-order FVE scheme holds at least $k$-(k-2)-order orthogonality. Recall the second row from the bottom of (4.9). 
Expand $u$ at $x_{0, K} \in K$, then

$$
\begin{aligned}
& \int_{K} p_{0, K} u^{\prime \prime}\left(\Pi_{h}^{1} w-\Pi_{h}^{k, *}\left(\Pi_{h}^{1} w\right)\right) d x \\
= & \int_{K} p_{0, K}\left(\sum_{i=0}^{k-1}\left(\frac{u^{(i+2)}\left(x_{0, K}\right)}{i !}\left(x-x_{0, K}\right)^{i}\right)+r_{K}^{\prime \prime}\right)\left(\Pi_{h}^{1} w-\Pi_{h}^{k, *}\left(\Pi_{h}^{1} w\right)\right) d x \\
= & \int_{K} p_{0, K}\left(\frac{u^{(k+1)}\left(x_{0, K}\right)}{(k-1) !}\left(x-x_{0, K}\right)^{k-1}+r_{K}^{\prime \prime}\right)\left(\Pi_{h}^{1} w-\Pi_{h}^{k, *}\left(\Pi_{h}^{1} w\right)\right) d x,
\end{aligned}
$$

where $r_{K}$ is the reminder of the $(k+1)$-order expansion. It's obvious that

$$
\sum_{K \in \mathcal{T}_{h}}\left|\int_{K} p_{0, K} r_{K}^{\prime \prime}\left(\Pi_{h}^{1} w-\Pi_{h}^{k, *}\left(\Pi_{h}^{1} w\right)\right) d x\right| \leq C h^{k+1}\|u\|_{k+2}\|w\|_{1} .
$$

However, when the $k$ - $\left(k\right.$-1)-order orthogonal condition is not satisfied, since $p_{0, K}$ $\times\left(u^{(k+1)}\left(x_{0, K}\right) /(k-1) !\right)$ is a constant on $K$, one cannot expect a higher-order estimate for the integral of $\left(x-x_{0, K}\right)^{k-1}\left(\Pi_{h}^{1} w-\Pi_{h}^{k, *}\left(\Pi_{h}^{1} w\right)\right)$ on $K$ by cancellation for arbitrary $w$. Generally, one only have

$$
\begin{aligned}
& \sum_{K \in \mathcal{T}_{h}} \int_{K} p_{0, K} \frac{u^{(k+1)}\left(x_{0, K}\right)}{(k-1) !}\left(x-x_{0, K}\right)^{k-1}\left(\Pi_{h}^{1} w-\Pi_{h}^{k, *}\left(\Pi_{h}^{1} w\right)\right) d x \\
= & \mathcal{O}\left(h^{k}\right)\|u\|_{k+1}\|w\|_{1} .
\end{aligned}
$$

Together (4.5) and (4.6),

$$
\begin{aligned}
\| u & -u_{h} \|_{0}^{2} \\
\geq- & \left|a\left(u-u_{h}, w-\Pi_{h}^{1} w\right)\right| \\
& -\left|\sum_{K \in \mathcal{T}_{h}} \int_{K}\left(\left(p-p_{0, K}\right)\left(u-u_{h}\right)^{\prime}\right)^{\prime}\left(\Pi_{h}^{1} w-\Pi_{h}^{k, *}\left(\Pi_{h}^{1} w\right)\right) d x\right| \\
& +\left|\sum_{K \in \mathcal{T}_{h}} \int_{K} p_{0, K}\left(\left(u-u_{h}\right)^{\prime}\right)^{\prime}\left(\Pi_{h}^{1} w-\Pi_{h}^{k, *}\left(\Pi_{h}^{1} w\right)\right) d x\right| \\
& \quad-\left|\sum_{K \in \mathcal{T}_{h}} \int_{K}\left(q\left(u-u_{h}\right)^{\prime}+r\left(u-u_{h}\right)\right)\left(\Pi_{h}^{1} w-\Pi_{h}^{k, *}\left(\Pi_{h}^{1} w\right)\right) d x\right| \\
\geq-C h^{k+1}\|u\|_{k+1}\|w\|_{2}-C h^{k+1}\|u\|_{k+1}\|w\|_{1} & +\mathcal{O}\left(h^{k}\right)\|u\|_{k+1}\|w\|_{1}-C h^{k+1}\|u\|_{k+1}\|w\|_{1} \\
=\mathcal{O} & \left(h^{k}\right)\|u\|_{k+1}\|w\|_{1}-C h^{k+1}\|u\|_{k+1}\|w\|_{2} .
\end{aligned}
$$

Recall, when we talk about the convergence order of a FVE scheme, it should generally work for any (2.1). Theoretically, it is possible to construct a pair of $\tilde{u}$ and $\tilde{u}_{h}$ such that

$$
C_{1}\|\tilde{w}\|_{2} \leq\|\tilde{w}\|_{1} \leq C_{2}\|\tilde{w}\|_{2}
$$


where $C_{1}$ and $C_{2}$ are independent to $h$ and $\tilde{u}_{h}$. For this $\tilde{u}$ and $\tilde{u}_{h}$, with the fact that $\|\tilde{w}\|_{2} \geq C\left\|\tilde{u}-\tilde{u}_{h}\right\|_{0}$, from (4.13), we further have

$$
\left\|\tilde{u}-\tilde{u}_{h}\right\|_{0} \geq \mathcal{O}\left(h^{k}\right)\|\tilde{u}\|_{k+1}
$$

That is to say, a $k$-order FVE scheme, which does not satisfy the $k$-(k-1)-order orthogonal condition, can hold at most $k$-order $L^{2}$ convergence rate, which meets with (4.11).

Remark 4.1. The proof of Theorems 4.1, 4.2 do not require the restriction $r-(1 / 2) q^{\prime} \geq$ $\gamma>0$ on the convection and reaction coefficients of the problem (2.1). If the stability and the optimal $H^{1}$ convergence rate can be ensured for some BVP (2.1) without satisfying $r-(1 / 2) q^{\prime} \geq \gamma>0$, the optimal $L^{2}$ convergence rate can also be ensured.

Remark 4.2. The analysis of Theorems 4.1, 4.2 are given element-wisely. Thus, defining the dual strategies of a FVE scheme by (3.2) element-wisely would not affects the process of the analysis. That is to say, the dual strategies in different primary elements could be different without affecting the $L^{2}$ convergence rate.

Remark 4.3. The conclusions are extensible to multi-dimensional tensorial meshes with different dual strategies in different directions. The numerical results for biquadratic/bicubic/biquatic FVE schemes over rectangular meshes are shown in Example 5.6 as an example. However, the theorem for multi-dimensional cases are not straightforward.

\section{Numerical experiments}

In this section, Example 5.1 shows the numerical results for some FVE schemes, which indicate that all FVE schemes hold optimal $H^{1}$ convergence rate, while only the FVE schemes satisfying the $k$ - $(k-1)$-order orthogonal condition possess the optimal $L^{2}$ convergence rate.

Example 5.2 shows the numerical $L^{2}$ convergence rates for quadratic FVE schemes and quartic FVE schemes (with symmetric dual). The numerical results well match with the solutions of the $k$ - $(k$-1)-order orthogonal condition. That is to say the $k$-(k-1)-order orthogonal condition is a sufficient and necessary condition for a FVE scheme to hold optimal $L^{2}$ convergence rate.

Example 5.3 shows the $L^{2}$ results for quadratic FVE schemes, who have different dual strategies in different primary elements. The dual strategy in each primary element satisfies the 2-1-order orthogonal condition respectively. The results illustrate that the dual strategies in different primary elements could be different.

Examples 5.4-5.5 compare the numerical performances of the standard quadratic FE scheme and quadratic FV schemes for three convection-dominated convection-diffusion problems. These two examples demonstrate the possibility and capacity of the FVE 
schemes (such as scheme FV2-6 in Example 5.4 and scheme FV2-7 in Example 5.5) with possibly asymmetric dual strategies to be used to solve some complex problems.

Example 5.6 shows the numerical $L^{2}$ results of three schemes, whose dual strategy are obtained from the tensor of the dual strategies of 1D cases, on rectangular meshes. All these schemes holds optimal $L^{2}$ convergence rate.

Example 5.1. Consider the BVP (2.1) with $p(x)=1, q(x)=x, r(x)=2, \Omega=[0, \pi]$, and $f$ is chosen such that the exact solution is $u(x)=\sin (x)$. Apply the FVE schemes listed in Table 1 to this problem. In which, schemes O- $k-i(k, i \leq 4)$ are $k$-order FVE schemes satisfying the $k$-(k-1)-order orthogonal condition, obtained by solving (3.1) with the corresponding $\mathbf{a}_{k}$. While, schemes $\mathrm{G}-k$ - $i$ do not satisfy the $k$-(k-1)-order orthogonal condition.

Here, we list in Table 2 the $H^{1}$ and $L^{2}$ results for schemes O-1-1 and G-1-1, in Fig. 2 the $H^{1}$ and $L^{2}$ results for schemes O-2-i and G-2-i $(i=1,2,3,4)$, and in Fig. 3 the $L^{2}$ results for schemes $\mathrm{O}-3 / 4-i$ and G-3/4-i $(i=1,2,3,4)$.

Table 1: The FVE schemes used in Example 5.1.

\begin{tabular}{||c|c|c|c|c|c||}
\hline & \multicolumn{3}{|c|}{ Orthogonal Schemes } & \multicolumn{2}{c||}{ General Schemes } \\
\hline$k$ & Schemes & $\vec{\alpha}_{k}$ & $\mathbf{a}_{k}$ & Schemes & $\vec{\alpha}_{k}$ \\
\hline 1 & O-1-1 & $1 / 2$ & - & G-1-1 & $1 / 3$ \\
\hline & O-2-1 & $(1 / 2-\sqrt{1 / 12}, 1 / 2+\sqrt{1 / 12})$ & $1 / 2$ & G-2-1 & $(1 / 3,2 / 3)$ \\
2 & O-2-2 & $(1 / 2-\sqrt{1 / 24}, 1 / 2+\sqrt{1 / 6})$ & $2 / 3$ & G-2-2 & $(1 / 2,9 / 16)$ \\
& O-2-3 & $(1 / 2-\sqrt{7 / 60}, 1 / 2+\sqrt{5 / 84})$ & $5 / 12$ & G-2-3 & $(1 / 4,5 / 13)$ \\
& O-2-4 & $(1 / 2-\sqrt{5 / 36}, 1 / 2+\sqrt{1 / 20})$ & $3 / 8$ & G-2-4 & $(1 / 3,4 / 7)$ \\
\hline \multirow{3}{*}{3} & O-3-1 & $(0.1797,0.4626,0.8127)$ & $(0.40,0.57)$ & G-3-1 & $(1 / 12,1 / 2,9 / 16)$ \\
& O-3-2 & $(0.1365,0.5636,0.9151)$ & $(0.33,0.78)$ & G-3-2 & $(1 / 7,1 / 3,5 / 9)$ \\
& O-3-3 & $(0.1018,0.4325,0.8521)$ & $(0.24,0.65)$ & G-3-3 & $(1 / 7,1 / 4,9 / 10)$ \\
& O-3-4 & $(0.1284,0.4937,0.8676)$ & $(0.30,0.69)$ & G-3-4 & $(1 / 11,2 / 3,3 / 4)$ \\
\hline \multirow{4}{*}{4} & O-4-1 & $(0.1312,0.1929,0.6264,0.9707)$ & $(0.23,0.37,0.86)$ & G-4-1 & $(1 / 5,2 / 5,3 / 5,4 / 5)$ \\
& O-4-2 & $(0.0457,0.3543,0.7767,0.9848)$ & $(0.15,0.58,0.93)$ & G-4-2 & $(1 / 6,2 / 5,1 / 2,3 / 4)$ \\
& O-4-3 & $(0.1153,0.4625,0.6335,0.9070)$ & $(0.28,0.58,0.77)$ & G-4-3 & $(1 / 9,1 / 4,1 / 3,1 / 2)$ \\
& O-4-4 & $(0.1197,0.4900,0.7395,0.9311)$ & $(0.29,0.66,0.83)$ & G-4-4 & $(1 / 7,2 / 7,4 / 7,6 / 7)$ \\
\hline
\end{tabular}

Table 2: Example 5.1.

\begin{tabular}{|c|c|c|c|c|c|c|c|c|}
\hline & \multicolumn{4}{|c|}{ Scheme O-1-1 } & \multicolumn{4}{|c|}{ Scheme G-1-1 } \\
\hline$h$ & $\overline{\left|u-u_{h}\right|_{1}}$ & Order & $\overline{\left\|u-u_{h}\right\|_{0}}$ & Order & $\left|u-u_{h}\right|_{1}$ & \begin{tabular}{|l|} 
Order \\
\end{tabular} & $\left\|u-u_{h}\right\|_{0}$ & Order \\
\hline $1 / 8$ & $1.4270 e-01$ & $\backslash$ & $8.3106 e-03$ & 1 & $1.4696 e-01$ & $\backslash$ & $4.7438 e-02$ & 1 \\
\hline $1 / 16$ & $7.1120 e-02$ & 1.0046 & $2.0702 e-03$ & 2.0051 & $7.4726 e-02$ & 0.9757 & $2.3279 e-02$ & 1.0270 \\
\hline $1 / 32$ & $3.5530 e-02$ & 1.0012 & $5.1692 e-04$ & 2.0018 & $3.7704 e-02$ & 0.9869 & $1.1579 e-02$ & 1.0075 \\
\hline $1 / 64$ & $1.7761 e-02$ & 1.0003 & $1.2918 e-04$ & 2.0006 & $1.8941 e-02$ & 0.9932 & $5.7805 e-03$ & 1.0023 \\
\hline $1 / 128$ & $8.8801 e-03$ & 1.0001 & $3.2291 e-05$ & 2.0002 & $9.4930 e-03$ & 0.9966 & $2.8887 e-03$ & 1.0008 \\
\hline
\end{tabular}




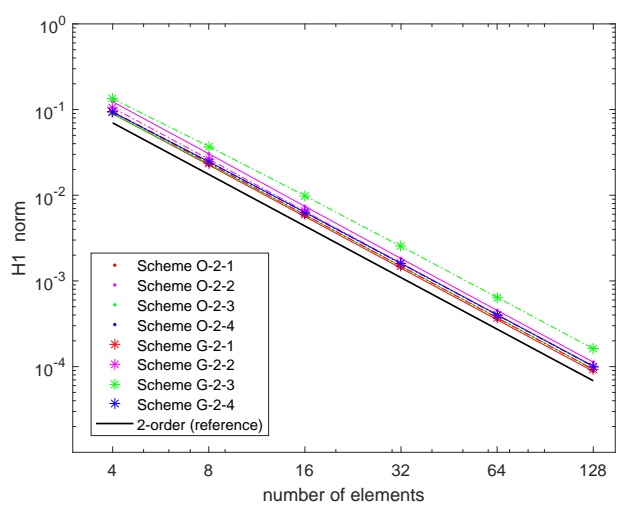

a) The $H^{1}$ convergence rate

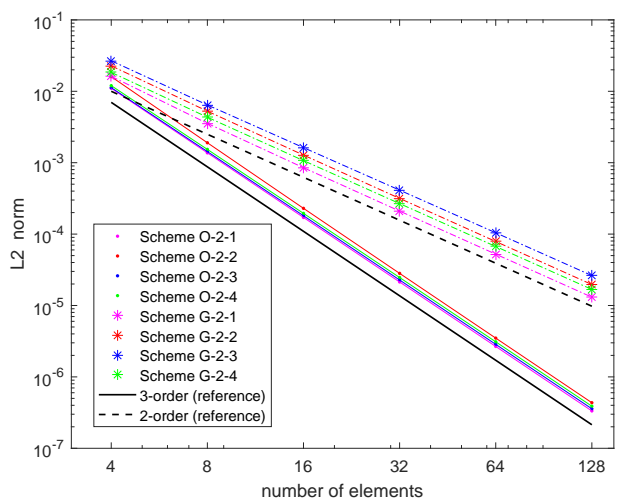

b) The $L^{2}$ convergence rate

Figure 2: The numerical results of the quadratic (2-order) FVE schemes.

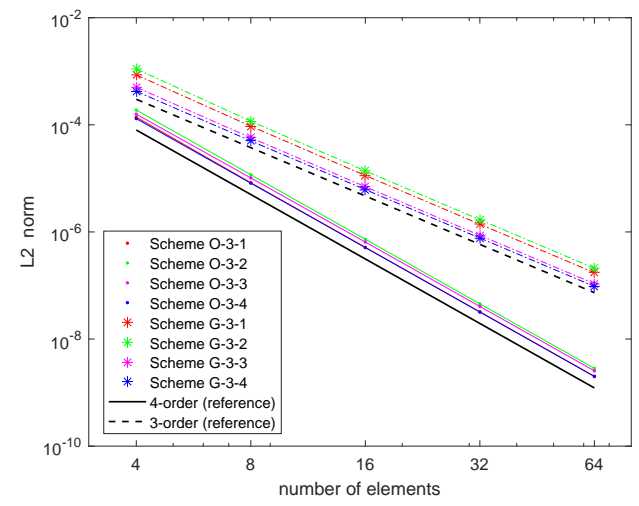

a) The cubic FVE schemes

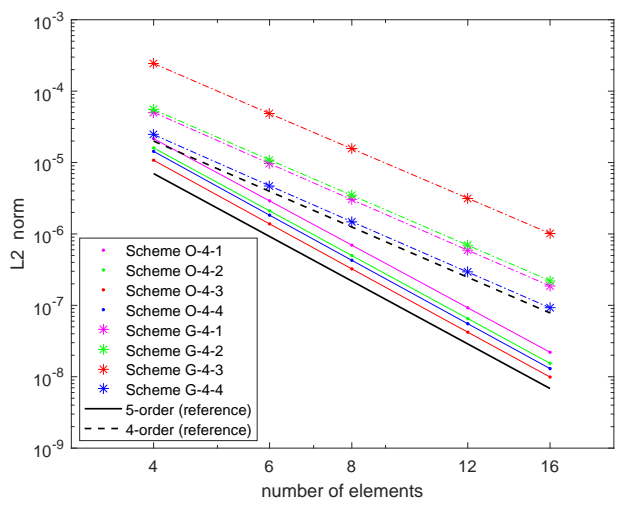

b) The quartic FVE schemes

Figure 3: The numerical $L^{2}$ results of the cubic (3-order) and quartic (4-order) FVE schemes.

Example 5.2. Apply the quadratic (2-order) FVEM and the quartic (4-order) FVEM to the BVP (2.1) with $p(x)=1, q(x)=r(x)=0$, and $f$ is chosen such that $u(x)=\sin x$. There are two independent variable parameters $\left(\alpha_{2}, \alpha_{1}\right) \in\left\{0<\alpha_{1}<\alpha_{2}<1\right\}$ for the quadratic FVEM, and two independent variable parameters $\left(\alpha_{4}, \alpha_{3}\right) \in\left\{1 / 2<\alpha_{3}<\right.$ $\left.\alpha_{4}<1\right\}$ for the quartic FVEM with symmetric dual strategies. Vary the parameters in the reasonable domains with step size $1 / 300$ respectively, and compute the discrete problem (2.3) over uniform primary meshes with mesh size $h=1 / 10$ and $h / 2$. Denote

$$
r_{L 2}=\frac{1}{\log (2)} \log \left(\frac{\left\|u-u_{h}\right\|_{0}}{\left\|u-u_{h / 2}\right\|_{0}}\right)
$$

as the numerical $L^{2}$ convergence rate of the FVE schemes.

Figs. 4(a) and 4(b) show the numerical $L^{2}$ convergence rate $r_{L 2}$ as functions of the parameters $\left(\alpha_{2}, \alpha_{1}\right)$ for the quadratic FVE schemes and $\left(\alpha_{4}, \alpha_{3}\right)$ for the quartic FVE 


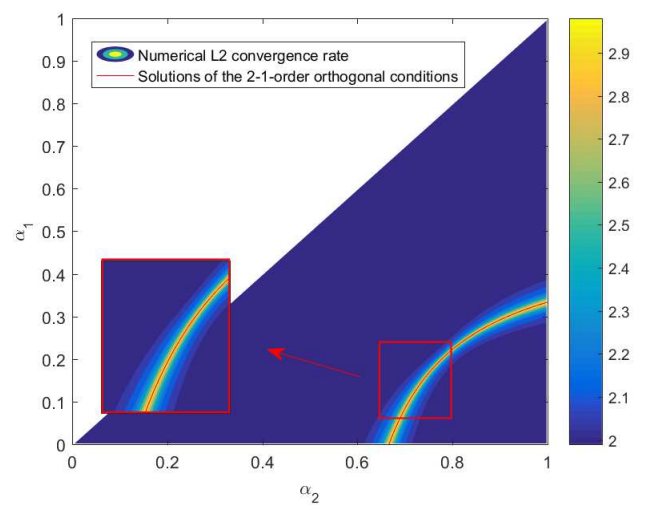

a) The quadratic FVEM

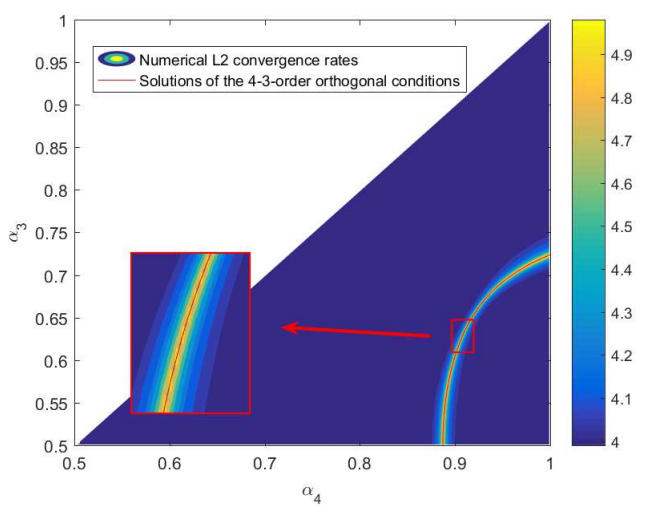

b) The symmetric quartic FVEM

Figure 4: Numerical $L^{2}$ convergence rate $r_{L 2}$ and the solutions of the orthogonal condition.

schemes, respectively. The red line is the solutions of the $k$ - $(k-1)$-order orthogonal condition (3.1). It shows that the region, where numerical $L^{2}$ convergence rate is close to optimal $(k+1)$-order, fits with the solutions of the red lines perfectly, which helps to verify that the $k$-(k-1)-order orthogonal condition is a necessary and sufficient condition for a FVE scheme to hold optimal $L^{2}$ convergence rate.

Example 5.3. Consider the quadratic FVE schemes with different dual strategies in different primary elements. The model problem is selected as the one in Example 5.1. The dual strategies in each primary elements of each schemes FV2-i $(i=1,2,3,4)$ for the first level $(h=1 / 4)$ are randomly selected from the solutions of (3.9). And, the dual strategies of the following levels are the same with their farther levels. We show in Table 3 the numerical $L^{2}$ results, which indicates that all these schemes hold optimal $L^{2}$ convergence rate.

Table 3: Example 5.3.

\begin{tabular}{||c|c|c|c|c|c|c|c|c||}
\hline & \multicolumn{2}{|c|}{ FV2-1 } & \multicolumn{2}{c|}{ FV2-2 } & \multicolumn{2}{c||}{ FV2-3 } & \multicolumn{2}{c||}{ FV2-4 } \\
\hline$h$ & $\left\|u-u_{h}\right\|_{0}$ & Order & $\left\|u-u_{h}\right\|_{0}$ & Order & $\left\|u-u_{h}\right\|_{0}$ & Order & $\left\|u-u_{h}\right\|_{0}$ & Order \\
\hline $1 / 4$ & $5.7866 e-03$ & $\backslash$ & $6.9984 e-03$ & $\backslash$ & $7.6671 e-03$ & $\backslash$ & $9.6704 e-03$ & $\backslash$ \\
$1 / 8$ & $7.6727 e-04$ & 3.0589 & $1.0340 e-03$ & 2.9908 & $1.0200 e-03$ & 2.9292 & $1.0817 e-03$ & 3.0573 \\
$1 / 16$ & $1.0008 e-04$ & 2.9926 & $1.2476 e-04$ & 2.9904 & $1.2910 e-04$ & 2.9602 & $1.3839 e-04$ & 3.0415 \\
$1 / 32$ & $1.0639 e-05$ & 3.0058 & $1.5600 e-05$ & 2.9937 & $1.6312 e-05$ & 2.9794 & $1.6401 e-05$ & 3.0210 \\
\hline
\end{tabular}

Example 5.4. Consider a convection-dominated problem that $p(x)=10^{-7}, q(x)=$ $1, r(x)=0$ in (2.1), $f$ is defined such that $u(x)=e^{x-1 / 2}$, and the boundary conditions are set as $u(0)=e^{-1 / 2}, u(1)=e^{1 / 2}$. Write FV2-5 the quadratic FVE scheme shares the same dual strategy with scheme O-2-1 in Table 1, and FV2-6 the quadratic FVE scheme 


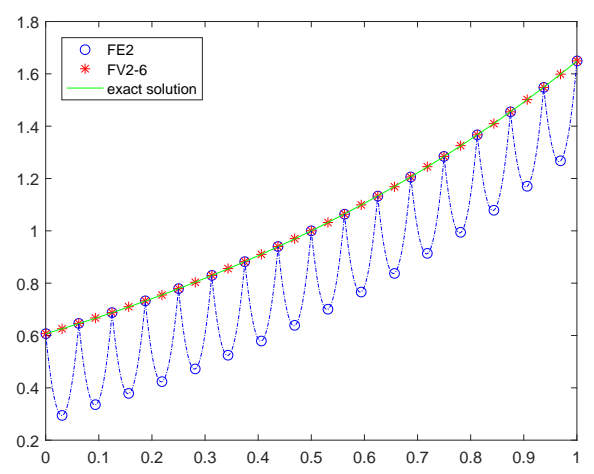

a) Numerical solutions of FE2 and FV2-6

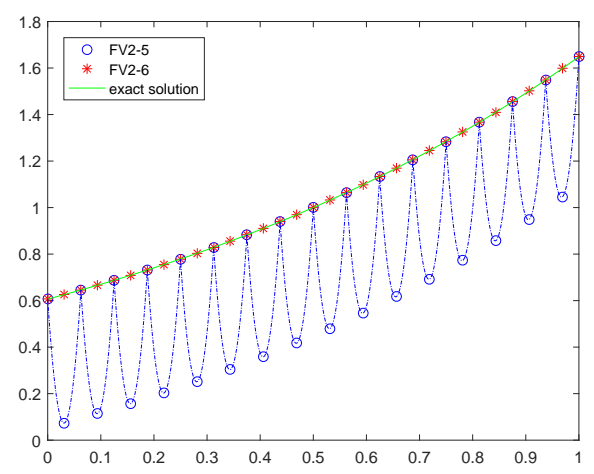

b) Numerical solutions of FV2-5 and FV2-6

Figure 5: The numerical results of Example 5.4.

with dual strategy $\left(\alpha_{1}, \alpha_{2}\right)=(0.0130,0.6711)$ (which is obtained by letting $a_{1}=0.26$ in (3.5)) in all primary elements.

Fig. 5 shows the numerical solutions when the mesh size is $h=1 / 16$, in which the solutions of the standard quadratic FE scheme (FE2) and FV2-5 have high numerical oscillation, while the solution of FV2-6 well fits the exact solution.

Example 5.5. Consider a variable coefficient convection-dominated problem that $p(x)=\exp (8 \sin (\pi(x-1 / 2))-8), q(x)=1, r(x)=0$ in (2.1), $f$ is defined such that $u(x)=e^{x}$, and the boundary conditions are set as $u(0)=1, u(1)=e$. The dual strategies of the quadratic FVE scheme FV2-7 are different in different primary elements. On $K$, it is given by $\left(\alpha_{1}, \alpha_{2}\right)$ obtained through letting $a_{1}=0.2501+p\left(x_{K}\right) / 4.1\left(x_{K}\right.$ is the midpoint of $K$ ) in (3.5). We still compare schemes FV2-7 with FE2 and FV2-6.

Fig. 6 shows that scheme FV2-7 has better convergence property than schemes FE2 and FV2-6 in this problem.

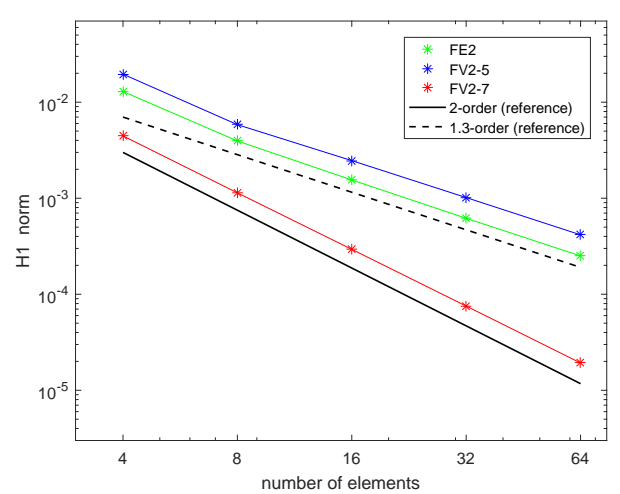

a) $H^{1}$ convergence rate

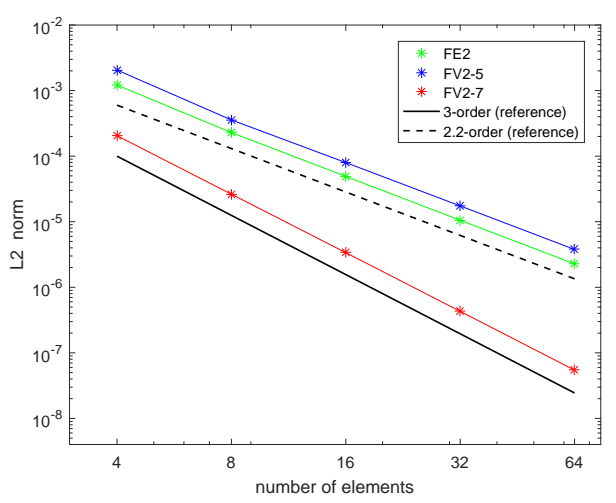

b) $L^{2}$ convergence rate

Figure 6: The numerical results of Example 5.5. 
Example 5.6. Consider the following elliptic problem on $\Omega=(0,1) \times(0,1)$ :

$$
\begin{cases}-\nabla \cdot(\mathbb{D} \nabla u)=f & \text { in } \Omega, \\ u=0 & \text { on } \partial \Omega\end{cases}
$$

with $\mathbb{D}=I . \quad f$ is selected such that $u=\sin (\pi x) \sin (\pi y)$. Construct the biquadratic FVE scheme FV2D-2 as the tensor of schemes O-2-1 (in $x$-direction) and O-2-2 (in $y$ direction) in Table 1, and the bicubic/biquartic FVE schemes FV2D-3/FV2D-4 as the tensor of schemes O-3-1/O-4-1 and O-3-2/O-4-2. Fig. 7(a) shows the dual elements of scheme FV2D-3, which is asymmetric in $y$-direction, and Fig. 7(b) shows that FV2D-2, FV2D-3 and FV2D-4 all have optimal $L^{2}$ convergence rate.

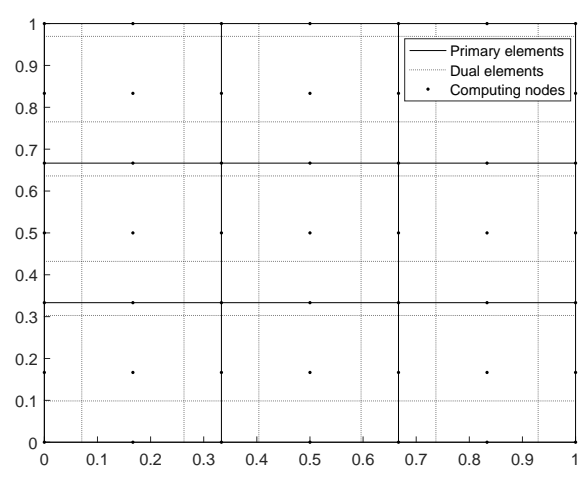

a) Dual meshes for FV2D-2

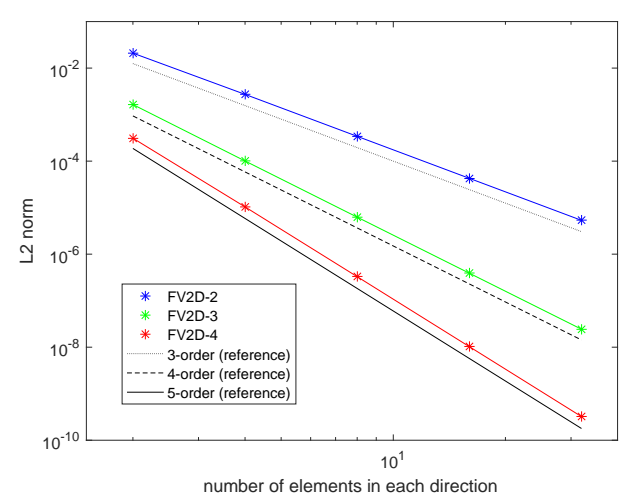

b) $L^{2}$ results for FV2D-2/3/4

Figure 7: The numerical results of Example 5.6.

\section{Conclusion}

The $k$-(k-1)-order orthogonal condition is proved to be a sufficient and necessary for a FVE scheme to have the optimal $L^{2}$ convergence rate. From the equivalent equations of the orthogonal conditions in Subsection 3.1, one can always construct a FVE scheme with optimal $L^{2}$ convergence rate. The dual strategies in different primary elements are not necessarily to be the same, and they are allowed to be asymmetric in each primary element. These opens up the possibilities and capacities of the FVEM to be applied to some complex problems, such as the convection-dominated problems. The construct method developed here is extensible to $2 \mathrm{D}$ problems over quadrilateral meshes, while the theory in 2D is not straightforward.

\section{Acknowledgement}

The authors thank the anonymous referees for their suggestions and comments which helped to improve the quality of this paper. This work was supported in part by the NSFC under grant No. 11701211. 


\section{Appendix A Stability and the proof of $H^{1}$ estimate}

The stability and $H^{1}$ estimate are the issues we can not skip when we study the $L^{2}$ estimate. The authors of $[4,27]$ gave some results for FVE schemes with some special dual strategies, such as the Gauss-Lobatto FVE schemes. In this section, we prove the stability and $H^{1}$ estimate for general FVE schemes. The proof in this section benefits a lot from the $k$-points numerical quadrature and reference [4].

We begin with some notations specially used in this section. Firstly, for all $w \in$ $H_{\mathcal{T}}^{m}(\Omega)$, where

$$
H_{\mathcal{T}}^{m}(\Omega):=\left\{w \in C(\Omega):\left.w\right|_{K_{i}} \in H^{m}\left(K_{i}\right), \forall K_{i} \in \mathcal{T}_{h}\right\},
$$

and all $j \geq 0$, we define a semi-norm and a norm by

$$
|w|_{j, \mathcal{T}}=\left(\sum_{K_{i} \in \mathcal{T}_{h}}|w|_{j, K_{i}}^{2}\right)^{1 / 2}, \quad\|w\|_{m, \mathcal{T}}=\left(\sum_{j=0}^{m}|w|_{j, \mathcal{T}}^{2}\right)^{1 / 2}
$$

Secondly, for any $v_{h}=\sum_{i=1}^{N} \sum_{j=1}^{k} v_{i, j} \psi_{i, j} \in V_{h}$, let

$$
\begin{aligned}
& \left|v_{h}\right|_{1, \mathcal{T}_{h}^{*}}^{2}=\sum_{i=1}^{N} \sum_{j=1}^{k} h_{i}^{-1}\left[v_{i, j}\right]^{2}, \quad\left\|v_{h}\right\|_{0, \mathcal{T}_{h}^{*}}^{2}=\sum_{i=1}^{N} \sum_{j=1}^{k} h_{i} v_{i, j}^{2}, \\
& \left\|v_{h}\right\|_{1, \mathcal{T}_{h}^{*}}^{2}=\left|v_{h}\right|_{1, \mathcal{T}_{h}^{*}}^{2}+\left\|v_{h}\right\|_{0, \mathcal{T}_{h}^{*}}^{2} .
\end{aligned}
$$

Noticing that $v_{1,0}=v_{N, k}=0$, it is easy to see the following Poincaré inequality

$$
\left\|v_{h}\right\|_{0, \mathcal{T}_{h}^{*}} \leq C\left|v_{h}\right|_{1, \mathcal{T}_{h}^{*}}, \quad \forall v_{h} \in V_{h},
$$

where the constant $C$ depends only on $\Omega$ and $k$.

Thirdly, we denote $A_{j}\left(j \in \mathbb{Z}_{k}\right)$ the weights of the $k$-points numerical quadrature

$$
Q_{k}(F)=\sum_{j=1}^{k} A_{j} F\left(\alpha_{j}\right)
$$

for computing the integral

$$
I(F)=\int_{-1}^{1} F(x) d x .
$$

The weights on $K_{i} \in \mathcal{T}_{h}$ are $A_{i, j}=h_{i} A_{j}, j \in \mathbb{Z}_{k}$. Then, we define a discrete semi-norm $|\cdot|_{1, \alpha}$ for all $w \in H_{0}^{1}(\Omega)$ by

$$
|w|_{1, \alpha}=\left(\sum_{i=1}^{N} \sum_{j=1}^{k} A_{i, j}\left(w^{\prime}\left(\alpha_{i, j}\right)\right)^{2}\right)^{1 / 2}
$$


Fourthly, a linear mapping $\Pi_{\mathcal{T}}^{*}: U_{h}^{k} \rightarrow V_{h}$ is given by ( $\Pi_{\mathcal{T}}^{*}$ is different from $\Pi_{h}^{k, *}$ defined in Subsection 2.3, and $\Pi_{\mathcal{T}}^{*}$ will be used only in this section)

$$
\Pi_{\mathcal{T}}^{*} w_{h}=\sum_{i=1}^{N} \sum_{j=1}^{k} w_{i, j} \psi_{i, j}, \quad w_{h} \in U_{h}^{k}
$$

where the coefficients $w_{i, j}$ are determined by the constraints

$$
\left[w_{i, j}\right]=A_{i, j} w_{h}^{\prime}\left(\alpha_{i, j}\right), \quad(i, j) \in \mathbb{Z}_{N} \times \mathbb{Z}_{k} \backslash\{(N, k)\},
$$

where $A_{i, j}$ s are the weights of $k$-points numerical quadrature on $K_{i}$. For $w_{h} \in U_{h}^{k}$, the derivative $\left.w_{h}^{\prime}\right|_{K_{i}} \in P^{k-1}\left(K_{i}\right), i \in \mathbb{Z}_{N}$, then

$$
\sum_{i=1}^{N} \sum_{j=1}^{k} A_{i, j} w_{h}^{\prime}\left(\alpha_{i, j}\right)=\int_{a}^{b} w_{h}^{\prime}(x) d x=w_{h}(b)-w_{h}(a)=0 .
$$

Therefore, recall $w_{1,0}=w_{N, k}=0$, then

$$
\begin{aligned}
& w_{N, k-1}=\sum_{(i, j) \neq(N, k)}\left[w_{i, j}\right] \\
= & \sum_{i=1}^{N} \sum_{j=1}^{k} A_{i, j} w_{h}^{\prime}\left(\alpha_{i, j}\right)-A_{N, r} w_{h}^{\prime}\left(\alpha_{N, k}\right)=-A_{N, r} w_{h}^{\prime}\left(\alpha_{N, k}\right) .
\end{aligned}
$$

In other words, we also have

$$
\left[w_{N, k}\right]=w_{N, k}-w_{N, k-1}=A_{N, k} w_{h}^{\prime}\left(\alpha_{N, k}\right) .
$$

According to the idea of the proof of Theorem 3.1 in [4], with the help of the $k$-points quadrature, we present the following lemma without the details of the proof.

Lemma A.1. Given an FVE scheme, for all $w_{h} \in U_{h}^{k}$, we have the equivalence property

$$
\left|\Pi_{\mathcal{T}}^{*} w_{h}\right|_{1, \mathcal{T}_{h}^{*}} \sim\left|w_{h}\right|_{1, \alpha} \sim\left|w_{h}\right|_{1, \mathcal{T}}
$$

Here, the semi-norms $|\cdot|_{1, \mathcal{T}_{h}^{*}},|\cdot|_{1, \alpha}$ and $|\cdot|_{1, \mathcal{T}}$ are given by (A.2), (A.4) and (A.1), respectively.

We are now ready to present the proof of the inf-sup condition in Theorem A.1.

Theorem A.1. For sufficiently small mesh size h, the following inf-sup condition are satisfied.

$$
\inf _{w_{h} \in U_{h}^{k}} \sup _{v_{h} \in V_{h}} \frac{a_{h}\left(w_{h}, v_{h}\right)}{\left\|w_{h}\right\|_{1}\left\|v_{h}\right\|_{\mathcal{T}_{h}^{*}}} \geq c_{0}
$$

where $c_{0}>0$ is a constant depending only on $k, \alpha_{0}, \kappa$ and $\Omega$. 
Proof. It follows from the bilinear form (2.3) that

$$
a_{h}\left(w_{h}, \Pi_{\mathcal{T}}^{*} w_{h}\right)=I_{1}+I_{2}, \quad \forall w_{h} \in U_{h}^{k}
$$

with

$$
\begin{aligned}
I_{1} & =\sum_{i=1}^{N} \sum_{j=1}^{k}\left[w_{i, j}\right] p\left(\alpha_{i, j}\right) w_{h}^{\prime}\left(g_{i, j}\right), \\
I_{2} & =\sum_{i=1}^{N} \sum_{j=1}^{k} w_{i, j} \int_{\alpha_{i, j}}^{\alpha_{i, j+1}}\left(q(x) w_{h}^{\prime}(x)+r(x) w_{h}(x)\right) d x .
\end{aligned}
$$

Therefore,

$$
I_{1} \geq p_{0} \sum_{i=1}^{N} \sum_{j=1}^{k} A_{i, j}\left(w_{h}^{\prime}\left(\alpha_{i, j}\right)\right)^{2} \sim p_{0}\left|w_{h}\right|_{1}^{2} .
$$

Next, we estimate $I_{2}$. Let

$$
V(x)=\int_{a}^{s}\left(q(s) w_{h}^{\prime}(s)+r(s) w_{h}(s)\right) d s
$$

and denote by

$$
E_{i}=\int_{x_{i-1}}^{x_{i}} w_{h}^{\prime}(x) V(x) d x-\sum_{j=1}^{k} A_{i, j} w_{h}^{\prime}\left(\alpha_{i, j}\right) V\left(\alpha_{i, j}\right)
$$

the error of the numerical quadrature in the in interval $\left[x_{i-1}, x_{i}\right], i \in \mathbb{Z}_{N}$. Then

$$
I_{2}=-\sum_{i=1}^{N} \sum_{j=1}^{k}\left[w_{i, j}\right] V\left(\alpha_{i, j}\right)=-\int_{a}^{b} w_{h}^{\prime}(x) V(x) d x+\sum_{i=1}^{N} E_{i} .
$$

With the fact that $w_{h}(a)=w_{h}(b)=0$ and

$$
\int_{a}^{b} q(x) w_{h}^{\prime}(x) w_{h}(x) d x=-\frac{1}{2} \int_{a}^{b} q^{\prime}(x) w_{h}^{2}(x) d x
$$

we obtain

$$
-\int_{a}^{b} w_{h}^{\prime}(x) V(x) d x=\int_{a}^{b}\left(r(x)-\frac{q^{\prime}(x)}{2}\right) w_{h}^{2}(x) d x \geq \gamma\left\|w_{h}\right\|_{0}^{2} .
$$

On the other hand, by (2.7.12) on page 98 of [12], for all $i \in \mathbb{Z}_{N}$

$$
E_{i}=\left(w_{h}^{\prime} V\right)^{(k)}\left(\xi_{i}\right) \mathcal{O}\left(h_{i}^{k+1}\right),
$$


where $\xi_{i} \in\left[x_{i-1}, x_{i}\right]$. By the Leibnitz formula for derivatives and the inverse inequality, we have

$$
\begin{aligned}
\left|\left(w_{h}^{\prime} V\right)^{(k)}\left(\xi_{i}\right)\right| & \leq \sum_{t=1}^{k}\left(\begin{array}{c}
k \\
t
\end{array}\right)\left|\left(q w_{h}^{\prime}+r w_{h}\right)^{(t-1)}\left(w_{h}^{\prime}\right)^{(k-t)}\left(\xi_{i}\right)\right| \\
& \leq c_{1} \sum_{t=1}^{k}\left\|w_{h}\right\|_{t, \infty, K_{i}}\left\|w_{h}\right\|_{(k-t+1), \infty, K_{i}} \\
& \lesssim c_{1} \sum_{t=1}^{k} h^{-(t-1 / 2)}\left|w_{h}\right|_{1, K_{i}} h^{-(k-t+1-1 / 2)}\left|w_{h}\right|_{1, K_{i}} \\
& =\tilde{c}_{1} h^{-k}\left|w_{h}\right|_{1, K_{i}}^{2}
\end{aligned}
$$

with

$$
c_{1}=\max \left\{\|q\|_{k-1, \infty, K_{i}},\|r\|_{k-1, \infty, K_{i}}\right\} \max _{t \leq k}\left(\begin{array}{c}
k \\
t
\end{array}\right) .
$$

Combining the estimates above, we have

$$
I_{2} \gtrsim \gamma\left\|w_{h}\right\|_{0, \mathcal{T}}^{2}-\tilde{c}_{1} h_{i}\left|w_{h}\right|_{1, \mathcal{T}}^{2}
$$

where $\tilde{c}_{1}$ is a constant independent of $h_{i}$. Then for sufficiently small $h$, we have

$$
a_{h}\left(w_{h}, \Pi_{\mathcal{T}}^{*} w_{h}\right) \geq \frac{p_{0}}{2}\left|w_{h}\right|_{1, \mathcal{T}}^{2}+\frac{\gamma}{2}\left\|w_{h}\right\|_{0, \mathcal{T}}^{2} \geq \frac{1}{2} \min \left\{p_{0}, \gamma\right\}\left\|w_{h}\right\|_{1, \mathcal{T}}^{2}
$$

Recall Lemma A.1, then we obtain

$$
\left\|w_{h}\right\|_{1, \mathcal{T}} \gtrsim\left\|\Pi_{\mathcal{T}}^{*} w_{h}\right\|_{\mathcal{T}_{h}^{*}}
$$

Therefore, for any $w_{h} \in U_{h}^{k}$,

$$
\sup _{v_{h} \in V_{h}} \frac{a_{h}\left(w_{h}, v_{h}\right)}{\left\|v_{h}\right\|_{\mathcal{T}_{h}^{*}}^{*}} \gtrsim \frac{a_{h}\left(w_{h}, \Pi_{\mathcal{T}}^{*} w_{h}\right)}{\left\|\Pi_{\mathcal{T}}^{*} w_{h}\right\|_{\mathcal{T}_{h}^{*}}} \geq c_{0}\left\|w_{h}\right\|_{1, \mathcal{T}},
$$

where $c_{0}$ is a constant depending only on $k, p_{0}, \gamma$, and $\Omega$. The inf-sup condition (A.6) then follows.

Through the inf-sup condition (A.6) and a similar procedure with [4], we have the $H^{1}$ estimate for FVE schemes with general dual meshes (which could be asymmetric).

Theorem A.2. Let $u \in H_{0}^{1}(\Omega) \cap H_{\mathcal{T}}^{k+1}(\Omega)$ and $u_{h} \in U_{h}$ be the solutions of (2.1) and (2.3), respectively.

$$
\left\|u-u_{h}\right\|_{1} \leq C h^{k}\|u\|_{k+1},
$$

where $C$ is a constant independent of $\mathcal{T}_{h}$ (or $h$ ). 


\section{References}

[1] R. E. BANK AND D. J. Rose, Some error estimates for the box method, SIAM Journal on Numerical Analysis, 24(4) (1987), 777-787.

[2] T. BARTh AND M. OhlBerger, Finite volume methods: Foundation and analysis, In E. Stein, R. d. Borst, and T. J. R. Hughes, editors, Encyclopedia of computational mechanics, volume 114, p. 45, Wiley, Chichester, 2004.

[3] Z. CAI, J. MANDEL, AND S. MCCORMICK, The finite volume element method for diffusion equations on general triangulations, SIAM Journal on Numerical Analysis, 28(2) (1991), 392-402.

[4] W. CAO, Z. ZHANG, AND Q. Zou, Superconvergence of any order finite volume schemes for $1 D$ general elliptic equations, Journal of Scientific Computing, 56(3) (2013), 566-590.

[5] W. CAO, Z. ZHANG, AND Q. ZOU, Is $2 k$-conjecture valid for finite volume methods?, SIAM Journal on Numerical Analysis, 53(2) (2015), 942-962.

[6] L. CHEN, A new class of high order finite volume methods for second order elliptic equations, SIAM Journal on Numerical Analysis, 47(6) (2010), 4021-4043.

[7] Z. Chen, R. LI, AND A. ZHou, A note on the optimal $L^{2}$-estimate of the finite volume element method, Advances in Computational Mathematics, 16(4) (2002), 291-303.

[8] Z. CHEN, J. WU, AND Y. XU, Higher-order finite volume methods for elliptic boundary value problems, Advances in Computational Mathematics, 37(2) (2012), 191-253.

[9] Z. Chen, Y. Xu, AND Y. ZHANG, A construction of higher-order finite volume methods, Mathematics of Computation, 84(292) (2015), 599-628.

[10] S.-H. CHOU AND X. YE, Unified analysis of finite volume methods for second order elliptic problems, SIAM Journal on Numerical Analysis, 45(4) (2007), 1639-1653.

[11] M. CUI AND X. YE, Unified analysis of finite volume methods for the stokes equations, SIAM Journal on Numerical Analysis, 48(3) (2010), 824-839.

[12] P. J. DAvis AND P. RABinowitz, Methods of Numerical Integration, Academic Press, Orland, 2nd edition, 1984.

[13] R. E. EWING, T. LIN, AND Y. LIN, On the accuracy of the finite volume element method based on piecewise linear polynomials, SIAM Journal on Numerical Analysis, 39(6) (2002), 1865-1888.

[14] F. FANG, Q. HONG, AND J. WU, Analysis of a special Q1-finite volume element scheme for anisotropic diffusion problems, Numerical Mathematics: Theory, Methods and Applications, 12(4) (2019), 1141-1167.

[15] Y. GAO, D. LIANG, AND Y. LI, Optimal weighted upwind finite volume method for convectiondiffusion equations in 2D, Journal of Computational and Applied Mathematics, 359 (2019), 73-87.

[16] W. HAсквUSCH, On first and second order box schemes, Computing, 41(4) (1989), 277296.

[17] J. HuANG AND S. XI, On the finite volume element method for general self-adjoint elliptic problems, SIAM Journal on Numerical Analysis, 35(5) (1998), 1762-1774.

[18] R. Li, Z. CHEN, AND W. Wu, Generalized Difference Methods for Differential Equations, Marcel Dekker, New York, 2000.

[19] R. LI AND X. ZHANG, A finite volume scheme for Savage-Hutter equations on unstructured grids, Numerical Mathematics: Theory, Methods and Applications, 13(2) (2020), 479496.

[20] Y. LI AND R. LI, Generalized difference methods on arbitrary quadrilateral networks, Journal of Computational Mathematics, 17(6) (1999), 653-672. 
[21] D. LIANG AND W. ZHAO, An optimal weighted upwinding covolume method on non-standard grids for convection-diffusion problems in $2 D$, International Journal for Numerical Methods in Engineering, 67(4) (2006), 553-577.

[22] F. LiEBAU, The finite volume element method with quadratic basis functions, Computing, 57(4) (1996), 281-299.

[23] Y. LIN, M. YANG, AND Q. Zou, $L^{2}$ error estimates for a class of any order finite volume schemes over quadrilateral meshes, SIAM Journal on Numerical Analysis, 53(4) (2015), 2030-2050.

[24] J. LV AND Y. LI, $L^{2}$ error estimates and superconvergence of the finite volume element methods on quadrilateral meshes, Advances in Computational Mathematics, 37(3) 2012, 393416.

[25] J. LV AND Y. LI, Optimal biquadratic finite volume element methods on quadrilateral meshes, SIAM Journal on Numerical Analysis, 50(5) (2012), 2379-2399.

[26] C. NIE, S. SHU, H. YU, AND W. XIA, Superconvergence and asymptotic expansions for bilinear finite volume element approximation on non-uniform grids, Journal of Computational and Applied Mathematics, 321 (2017), 323-335.

[27] M. PleXOUSAKIS AND G. E. ZOURARIS, On the construction and analysis of high order locally conservative finite volume-type methods for one-dimensional elliptic problems, SIAM Journal on Numerical Analysis, 42(3) (2004), 1226-1260.

[28] T. SCHMidT, Box schemes on quadrilateral meshes, Computing, 51(3-4) (1993), 271-292.

[29] S. SU AND J. WU, A vertex-centered and positivity-preserving finite volume scheme for twodimensional three-temperature radiation diffusion equations on general polygonal meshes, Numerical Mathematics: Theory, Methods and Applications, 13(1) (2020), 220-252.

[30] E. SÜLI, Convergence of finite volume schemes for poisson's equation on nonuniform meshes, SIAM Journal on Numerical Analysis, 28(5) (1991), 1419-1430.

[31] X. WANG, W. HuANG, AND Y. LI, Conditioning of the finite volume element method for diffusion problems with general simplicial meshes, Mathematics of Computation, 88(320) (2019), 2665-2696.

[32] X. WANG AND Y. LI, $L^{2}$ error estimates for high order finite volume methods on triangular meshes, SIAM Journal on Numerical Analysis, 54(5) (2016), 2729-2749.

[33] X. WANG AND Y. LI, Superconvergence of quadratic finite volume method on triangular meshes, Journal of Computational and Applied Mathematics, 348 (2019), 181-199.

[34] H. XIE, C. ZhaI, X. Xu, PEng Jun, AND H. Yong, A monotone finite volume scheme with fixed stencils for $3 D$ heat conduction equation, Communications in Computational Physics, 26(4) (2019), 1118-1142.

[35] J. XU AND Q. Zou, Analysis of linear and quadratic simplicial finite volume methods for elliptic equations, Numerische Mathematik, 111(3) (2009), 469-492.

[36] M. YANG, J. LIU, AND Y. LIN, Quadratic finite-volume methods for elliptic and parabolic problems on quadrilateral meshes: optimal-order errors based on barlow points, IMA Journal of Numerical Analysis, 33(4) (2013), 1342-1364.

[37] Z. ZHANG AND Q. ZOU, Vertex-centered finite volume schemes of any order over quadrilateral meshes for elliptic boundary value problems, Numerische Mathematik, 130(2) (2015), 363-393. 